\title{
Neurophysiological and neuropsychological damages produced by cocaine in adult populations
}

\begin{abstract}
This preliminary study shows that cocaine addiction is a public health problem. We seek to present a model of transdisciplinary approach to understand addictions through this analysis.
\end{abstract}

Keywords: addiction, psychotropic effects, behavioral alterations, depression, cocaine, neurophysiological, transdisciplinary

\author{
Volume 5 Issue 3 - 2018 \\ Nicolás Parra-Bolaños,' Juan Sebastián \\ Benjumea-Garcés,' Sindy Yuliana Gallego- \\ Tavera $^{2}$ \\ 'GRIESO Research Group, Institución Universitaria Marco Fidel \\ Suárez, Colombia \\ ${ }^{2}$ GRINSES Research Group - Fundapampuri ONG, Universidad \\ Católica Luis Amigó, Colombia
}

\begin{abstract}
Correspondence: Nicolás Parra-Bolaños, Professor and Researcher, GRIESO Research Group, Institución Universitaria Marco Fidel Suárez, Street 48, 50-30 Bello, Colombia, Tel +5744601505, +57-3128385534, Email inv.nbolanos@iumafis.edu.co
\end{abstract}

Received: April 21, 2018 | Published: May 07, 2018
Abbreviations: CASP, critical appraisal skills program; STROBE, strengthening the reporting of observational studies in epidemiology

\section{Introduction}

Cocaine is one of the most consumed drugs around the world. This drug is a very powerful chemical that has combinations and double effect. In the first place, it produces behavioral alterations and, secondly, it produces neurological alterations. Cocaine is an illegal product that is usually exported by South American countries to Europe and North America. ${ }^{1}$ The spread of cocaine gained popularity in these two regions of the world from the 1980s to 1990s. Cocaine produces vasodilation and generates visual, perceptual, motor and cardiovascular alterations in its initial phases. Dopamine is one of the neurotransmitters that increase activation during the first hours after consuming cocaine. ${ }^{2}$ This drug differs from many others in that it has the ability to create deposits within the brain of regular consumers. These deposits are the remains of cocaine that are usually lodged in subcortical regions of the brain, making it much more difficult to eliminate them. ${ }^{3}$ When the excitement phase produced by cocaine begins to decrease, a central nervous system depression effect occurs. This depression causes that they begin to produce addictive behaviors and force the consumer to look for contexts of easy access to this type of illegal drugs. ${ }^{4}$

\section{Methodology}

A partial search was made of the terms of the title of this research. We have tried to make a methodical and rigorous selection of databases known as Pubmed, Scopus, Web of Science and Index Medicus. The Critical Appraisal Skills Program - CASP is being used and as a backup to compare the data, we will continue using the Strengthening the Reporting of Observational Studies in Epidemiology - STROBE, which are a fundamental tool to verify the rigor of our study. We compared models of the most relevant contemporary authors of neurophysiology and neuropsychology for the construction of this manuscript.

\section{Discussion}

Cocaine is a highly addictive chemical, which is diluted along with other industrial components such as levamisole or hydroxyzine, which increase the psychotropic effects of this drug. ${ }^{5}$ The cardiovascular system is one of the most harmful physiological systems for consumers, because the heartbeat is accelerated excessively and harmful to health ${ }^{6}$ Cocaine is highly stimulant and has hallucinogenic effects, which puts the consumer's life in serious risk, since it causes it to be exposed to high-risk situations due to the search for dangerous contexts. This drug alters the response of the reward systems of the brain, causing conditioning to occur by associative learning to cocaine in an exacerbated manner. ${ }^{7}$ Glutamatergic systems are also altered by cocaine use. Glutamate is a neurotransmitter of excitatory functions, which is damaged by the habitual consumption of cocaine. Cocaine consumption already has pandemic characteristics, due to its powerful excitatory effects on the central nervous system, causing that when there is a strong dependence on this drug, relapses oblige the consumer to engage in behaviors that damage their family environment, their emotional environment, their work environment and their social environment to achieve them. ${ }^{8}$

Neural communication known as synapsis occurs by two modalities: excitation or inhibition, which is severely altered in cocaine use. If the functional connectivity in the brain is altered, the basic and superior psychological processes will obviously be altered. ${ }^{9}$ The alterations produced by cocaine include executive functions of the brain such as memory, motivation, emotional states, attentional processes, perception, language, thinking, cognition, sensations and oculo-motor systems. ${ }^{10}$ All new learning produces modifications in the cerebral cytoarchitecture, which is very notorious with the consumption of illegal drugs, as they damage the neurophysiological processes and the neuroanatomy of the brain. Cocaine use affects the brain in the short term, medium term and long term. This is due to the fact that cocaine deposits generated within subcortical regions are not eliminated over the years. ${ }^{11}$ Hallucinations are one of the most persistent neurological damages in users of cocaine, which makes populations addicted to this drug, are at risk of suffering serious 
accidents by crossing moments when they do not distinguish between reality and hallucinations. The dopaminergic systems are affected by cocaine consumption from the first time this drug is consumed, which is an indication of the negative consequences of cocaine on the body ${ }^{12}$.

Quality of life is one of the most quantified aspects to demonstrate the alterations produced by cocaine in adult consumers. The health psychology together with clinical neuropsychology have specialized in identifying the psychosocial aspects that are cracked with the habitual consumption of cocaine in adults. ${ }^{13}$ These two scientific fields have found that cocaine begins by damaging several cognitive processes in the habitual consumer and then begins to weaken the family and social support networks. Health psychology has identified that the quality of life and the perception of quality of life in neophyte cocaine users does not show differences in the mentality of the consumers, which means that they have a hard time understanding that they are beginning to suffer neurophysiological changes and severe behavioral changes. ${ }^{14}$ On the other hand, clinical neuropsychology has found that it is necessary to design neuropsychological tests specifically for consumers of cocaine and its derivatives. This is necessary because every functional damage must be tracked in neuropsychological aspects in the different stages of the evolution of the illegal drug consumption. ${ }^{15}$ The small cognitive and executive changes that adult cocaine users present from the first time must be measured with neuropsychological batteries that can quantify these changes and compare them with other stages of consumption. ${ }^{16}$

Mortality rates in adult populations consuming cocaine are up to $15 \%$ higher than the rest of the world population. While the risks of stroke increases by up to $6 \%$, which means that the consumption of cocaine puts people's lives at high risk. Men have higher rates of risk of death from cocaine use in relation to women. ${ }^{17}$ This is because adult males have a higher risk of heart attacks and consume higher doses of cocaine in search of adrenaline and altered states of consciousness. The adverse effects of cocaine use are clearly seen in the cardiovascular system, since they produce significant increases in heart rate, generate alterations in blood pressure and adversely affect the contractility of the left ventricle of the heart. ${ }^{18}$ Cocaine use is also correlated with the early appearance of clinical signs of thrombosis and arrhythmias. Clinical neuropsychology offers great possibilities to understand the cognitive changes that executive functions suffer in adult cocaine users, so it is necessary that neuropsychologists can perform joint work with other specialists to intervene in addictions. ${ }^{19}$ Health sciences should build bridges to bring applied sciences and basic sciences that work with adult cocaine users. This joint work is essential to prevent occasional users of cocaine from becoming habitual consumers. It is necessary to create tests in work, sports, cultural and educational environments to detect early cocaine use. ${ }^{20}$

\section{Conclusion}

The encephalic regions altered by cocaine consumption, ranging from subcortical areas to neocortical areas. Several neurotransmitters are affected and altered by cocaine, not only during times of consumption, but also in the days and weeks after consumption. Early contextual evaluation from the Experimental Analysis of Behavior should be one of the most used tools to prevent the consumption of cocaine in adult populations. Contexts are the starting point to scientifically understand cocaine consumption behaviors, since we must not only have a neuroscientific approach, but from experimental psychology and comparative psychology. The behavioral sciences linked to the health sciences, both in stages prior to the consumption of cocaine and in stages of habitual consumption, must be unified in a transdisciplinary way to provide comprehensive solutions to avoid cocaine consumption or at least decrease that social problem. It is suggested the creation of integrative models between neuropsychology, neurophysiology, social work, citizen care and family therapy to strengthen the support networks in potential adult populations consuming cocaine. Finally, the generation of educational and psychosocial spaces based on schedules of reinforcement and contextual contingencies is recommended to extinguish illegal drug consumption behaviors. Our scientific opinion is that neuropsychological rehabilitation programs should be generated in adults addicted to cocaine to improve the neurophysiology of these individuals.

\section{Acknowledgements}

The authors gratefully acknowledge their dear colleague Julian Alejandro Gaviria Lopez, PhD of the Neurology and Imaging of Cognition Lab (Labnic), University of Geneva, Campus Biotech (Switzerland) for their scientific support and contributions to this study.

\section{Conflict of interest}

The author declares that there is no conflict of interests regarding the publication of this paper.

\section{References}

1. Pena-Bravo J, Reichel C, Lavin A. Abstinence from Cocaine-Induced Conditioned Place Preference Produces Discrete Changes in Glutamatergic Synapses onto Deep Layer 5/6 Neurons from Prelimbic and Infralimbic Cortices. eNeuro. 2017;4(6):8-17.

2. Kang B, Song S, Wen L, et al. Effect of optogenetic manipulation of accumbal medium spiny neurons expressing dopamine D2 receptors in cocaineinduced behavioral sensitization. Eur J Neurosci. 2017;46(4):2056-2066.

3. Burton AC, Bissonette GB, Zhao AC, et al. Prior Cocaine SelfAdministration Increases Response-Outcome Encoding That Is Divorced from Actions Selected in Dorsal Lateral Striatum. J Neurosci. 2017;37(32):7737-7747.

4. Taugher RJ, Lu Y, Fan R, et al. ASIC1A in neurons is critical for fearrelated behaviors. Genes Brain Behav. 2017;16(8):745-755.

5. Anderson E, Self D. It's only a matter of time: longevity of cocaineinduced changes in dendritic spine density in the nucleus accumbens. Curr Opin Behav Sci. 2017;13:117-123.

6. Mills F, Globa AK, Liu S, et al. Cadherins mediate cocaine-induced synaptic plasticity and behavioral conditioning. Nat Neurosci. 2017;20(4):540-549.

7. Otis J, Mueller D. Reversal of Cocaine-Associated Synaptic Plasticity in Medial Prefrontal Cortex Parallels Elimination of Memory Retrieval. Neuropsychopharmacology. 2017;42(10):2000-2010.

8. Dos Santos M, Salery M, Forget B, et al. Rapid Synaptogenesis in the Nucleus Accumbens Is Induced by a Single Cocaine Administration and Stabilized by Mitogen-Activated Protein Kinase Interacting Kinase-1 Activity. Biol Psychiatry. 2017;82(11):806-818.

9. Ruan H, Yao W. Cocaine Promotes Coincidence Detection and Lowers Induction Threshold during Hebbian Associative Synaptic Potentiation in Prefrontal Cortex. J Neurosci. 2017;37(4):986-997. 
10. Gutman AL, Nett KE, Cosme CV, et al. Extinction of Cocaine Seeking Requires a Window of Infralimbic Pyramidal Neuron Activity after Unreinforced Lever Presses. J Neurosci. 2017;37(25):6075-6086.

11. Zhang L, Huang L, Lu K, et al. Cocaine-induced synaptic structural modification is differentially regulated by dopamine D1 and D3 receptorsmediated signaling pathways. Addict Biol. 2017;22(6):1842-1855.

12. Liu X, Zhong P, Vickstrom C, et al. PDE4 Inhibition Restores the Balance Between Excitation and Inhibition in VTA Dopamine Neurons Disrupted by Repeated In Vivo Cocaine Exposure. Neuropsychopharmacology. 2017;42(10):1991-1999.

13. Blacktop JM, Todd RP, Sorg BA. Role of perineuronal nets in the anterior dorsal lateral hypothalamic area in the acquisition of cocaine-induced conditioned place preference and self-administration. Neuropharmacology. 2017; 118:124-136.

14. Singer BF, Bryan MA, Popov P, et al. Rapid induction of dopamine sensitization in the nucleus accumbens shell induced by a single injection of cocaine. Behav Brain Res. 2017;324:66-70.

15. DePoy LM, Zimmermann KS, Marvar PJ, et al. Induction and Blockade of Adolescent Cocaine-Induced Habits. Biol Psychiatry. 2017;81(7):595605

16. Li X, Peng XQ, Jordan CJ, et al. mGluR5 antagonism inhibits cocaine reinforcement and relapse by elevation of extracellular glutamate in the nucleus accumbens via a CB1 receptor mechanism. Sci Rep.
2018;8(1):3686

17. Beaudoin GMJ, Gomez JA, Perkins J, et al. Cocaine Selectively Reorganizes Excitatory Inputs to Substantia Nigra Pars Compacta Dopamine Neurons. J Neurosci. 2018;38(5):1151-1159.

18. Ingebretson AE, Hearing MC, Huffington ED, et al. Endogenous dopamine and endocannabinoid signaling mediate cocaine-induced reversal of AMPAR synaptic potentiation in the nucleus accumbens shell. Neuropharmacology. 2018;131:154-165.

19. James MH, McGlinchey EM, Vattikonda A, et al. Cued Reinstatement of Cocaine but Not Sucrose Seeking Is Dependent on Dopamine Signaling in Prelimbic Cortex and Is Associated with Recruitment of Prelimbic Neurons That Project to Contralateral Nucleus Accumbens Core. Int $J$ Neuropsychopharmacol. 2018;21(1):89-94.

20. Guillem K, Ahmed SH. Preference for Cocaine is Represented in the Orbitofrontal Cortex by an Increased Proportion of Cocaine Use-Coding Neurons. Cereb Cortex. 2018;28(3):819-832. 